\title{
Notes
}

\section{Decoding back-projected slides by means of photocell-operated logic}

WILLIAM S. BEAMON and E. SCOTT GELLER,' VIRGINIA POIYTECHNIC INSTITUTE, Blacksburg, Virginia 2406I

Many human decision-making, concept-formation, and verbal-learning experiments utilize back-projected slides to present information to Ss. Following each slide presentation, Ss are typically required to process the information and make a decision response. The following instrumentation technique may be used to give Ss automatic feedback following their response to each slide presentation.

The stimulus slides are coded by making a certain portion of the lower slide area transparent (e.g., see slide area No. 3 of Fig. 1). The specific location of the transparent area on each slide corresponds to the particular classification of that slide; the other area along the bottom of the slide remains opaque. The Ss' side of the projection screen is masked so that the bottom of each slide (i.e., coded area) cannot be seen. Photocells are placed along the bottom of the projection screen so that light will activate these photocells according to the coding logic. Each photocell operates a specific relay by means of a two-transistor Darlington Pair amplifier which provides a high degree of sensitivity (Fig. 2). The relay may then operate a clock, turn on a light, or be combined with $\mathrm{Ss}$ ' specific response through relay logic to give Ss automatic response feedback. This technique of decoding slide information has been found to be relatively inexpensive, trouble free, and quite versatile.

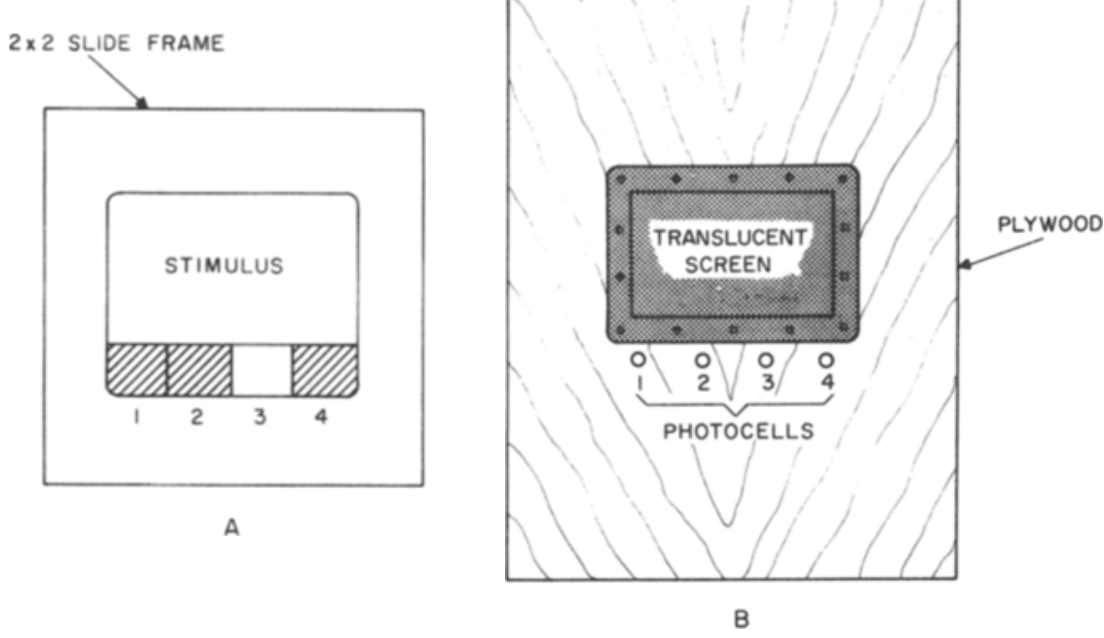

Fig. 1. Slide coding and photocell location. (The slide is projected on the screen side illustrated and Ss view the stimulus on the opposite side of the transiucent screen.)

Table 1

List of the Electronic Components in Fig. 2.

PC1: Photocell. Clairex CLSM5L. Price- $\$ 1.50$. Allied Part No. 60D6443.

R1: Resistor, 2,200 ohms, $1 / 2 \mathrm{~W}, 10 \%$ Price-\$.12, Allied No. 45D6040C

R2: Potentiometer, $50 \mathrm{~K}$ ohms, $1 \mathrm{~W}$. Price-\$1.02, Allied No. 46D3763.

C1: Capacitor, .005 mF, 1,000 V. Price- $\$ .24$ Allied No. 43D4244.

Q1: Transistor, NPN 2N3826 (Texas Instruments). Price-\$.54, Allied No. 2 N3826.

Q1: Transistor, NPN 40346 (RCA). Price-\$1.14, Allied No. 40346.

K1: Relay, 24 NDC, 4 PDT, Potter \& Brumfield. Price-\$3.65, Allied No.41D5785, Type MTP 17 D11 (K1 may be a standard panel-mounted relay, in which case the transistors would be connected to the operate contact rather than the lower side of the relay as shown). $\$ 8.21$ per amplifier unit.

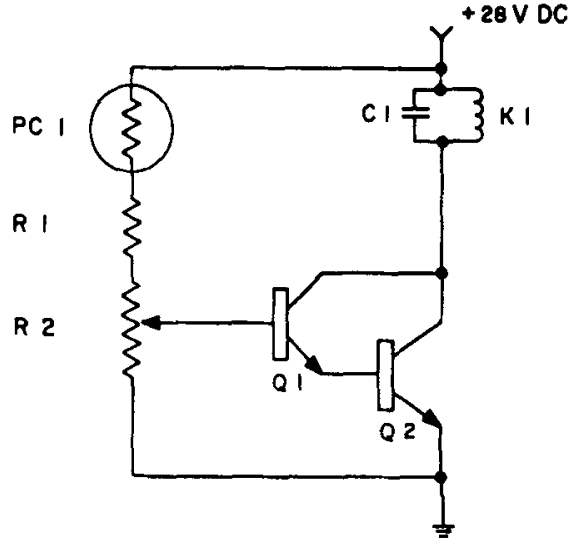

Fig. 2. Amplifier schematic. (Refer to Table 1 for a list of the components.)

\section{NOTE}

1. Requests for reprints should be sent to $E$ Scott Geller, Department of Psychology, Virginia Poly technic Institute, Blacksburg, Virginia 24061 